Proceedings of $\mathrm{CHT}-15$

ICHMT International Symposium on Advances in Computational Heat Transfer

May 25-29, 2015, Rutgers University, Piscataway, USA

CHT-15-222

\title{
THREE-DIMENSIONAL CONVECTION WITHIN A CUBOIDAL POROUS CAVITY DUE TO UNIFORM VOLUMETRIC HEATING
}

\author{
D. A. S. Rees \\ Department of Mechanical Engineering, University of Bath, \\ Claverton Down, Bath BA2 7AY, United Kingdom. \\ Email: D.A.S.Rees@bath.ac.uk
}

\begin{abstract}
A cuboidal cavity is filled with a fluid-saturated porous medium which is subjected to a uniform volumetric internal heating where all six boundaries are held at identical constant temperatures. The ensuing free convection is computed using finite difference approximations of the equations written using the velocity-potential/temperature formulation. Both steady and unsteady solvers have been written, and both employ a Full Approximation Scheme multigrid methodology with pointwise Gauss-Seidel smoothing. Our aim is to determine the evolution with Darcy-Rayleigh number, $R a$, of the flow and temperature fields within the cavity and how they undergo successive transformations from a steady single-cell pattern at relatively low values of $R a$ via symmetry-breaking bifurcations to more complicated states. The ensuing free convective flow is visualised by means of heat transfer contours and horizontal velocity vectors at the upper surface. In this preliminary work, attention is confined to a $2 \times 2 \times 1$ cavity and Darcy-Rayleigh numbers which are below 6000. Nevertheless we uncover a variety of competing steady-state solutions.
\end{abstract}

\section{INTRODUCTION}

Natural convection in enclosures which are filled with a fluid-saturated porous medium has occupied the centre stage in many fundamental heat transfer analyses, and the research literature is replete with papers which discuss a huge variety of different types of flow. On the other hand, natural convection which is induced by internal heat generation has been considered by only a few even though it arises in various physical problems such as heat removal from fuel debris in nuclear reactors, the underground disposal of radioactive waste materials, and exothermic chemical reactions in packed-bed reactors. Vasseur et al. [1984] presented numerical solutions for the problem of free convection in a porous layer bounded by two horizontal concentric cylinders with uniformly distributed energy sources. Prasad [1987], on the other hand, has studied numerically the steady free convection in a vertical rectangular cavity filled with a heat-generating saturated porous medium where the vertical walls of the cavity are isothermally cooled and the horizontal walls are adiabatic. An analysis of steady convection at asymptotically large Rayleigh numbers in a rectangular cavity was undertaken by Blythe et al [1985] and boundary layers were found to exist near the vertical sidewalls. Linear stability criteria in terms of the external and internal Rayleigh numbers were calculated by Gasser and Kazimi [1976] for the case of impermeable conducting boundaries, where the external Rayleigh number is based on an overall temperature drop across the layer. More recently, Nouri-Borujerdi et al. [2007], in a study of the effects of local thermal nonequilibrium, have quoted a value of $R a_{c}=471.3787$ as the critical Darcy-Rayleigh number above which convection ensues in an infinitely wide layer.

Nonlinear thermal convection in an infinite horizontal porous layer with internal heat sources was studied by Tveitereid [1977]. Three different steady flows were analysed: down-hexagons, up-hexagons 
and two-dimensional rolls. The stability of the flows with respect to small disturbances was investigated. It was found that down-hexagons are stable for Rayleigh numbers $R a$ up to 8 times the critical value $\left(8 R a_{c}\right)$, while up-hexagons are unstable for all values of $R a$. Moreover, two dimensional rolls are found to be stable in the range $3 R a_{c}<R a<7 R a_{c}$. His computed $N u$ versus $R a$ curves correlate quite well with the upper branch of Buretta and Berman's [1976] curve, even though these authors conducted experiments in cylindrical geometries.

Other papers on this general theme include the effect of saturating the porous medium with a nanofluid (Nield and Kuznetsov [2013]), and this tends to reduce the critical Darcy-Rayleigh number for the onset of convection. Bhadauria et al. [2013] consider the effect of applying g-jitter on the onset of convection while Mealey and Merkin [2009] employ a heat generation model whose strength is proportional to a power of the difference between the local and the ambient temperatures.

As far as we are aware, apart from the above-quoted study by Tveitreid [1977] which is in an unbounded layer, no computations of three-dimensional convection in enclosed cavities have been published to date. Therefore this paper forms a study of three-dimensional natural convection in a cuboidal enclosure which is bounded by six rigid and impermeable walls each of which are maintained at the same constant temperature, and which contain s a heat-generating fluid-saturated porous medium. Although this appears to be a very simple flow configuration, little is known about how the flow evolves with Rayleigh number in finite cavities. The paper by Banu et al. [1998] is the nearest there is to the present problem because it is concerned with the analogous two-dimensional cavity. These authors found (i) that flow arises at all non-zero values of the Darcy-Rayleigh number, (ii) that the first change in behaviour as the Darcy-Rayleigh number rises is a bifurcation to an unsteady convection pattern, and (iii) that the flow soon enters a chaotic state, particularly for shallow cavities.

We focus attention here on the $2 \times 2 \times 1$ cavity (i.e. one with a square planform and unit nondimensional height) and determine how the flow changes as Ra increases. This is undertaken by using a combination of steady and unsteady numerical solvers which are based upon a velocity-potential/temperature formulation of the governing equations and upon a full approximation scheme multigrid algorithm which is applied to a second order accurate finite difference discretization.

\section{NOMENCLATURE}

$A_{x}, A_{y}$
$C$
$g$
$h$
$k$
$K$
$N u$
$p$
$q^{\prime \prime \prime}$
$\operatorname{Ra}$
$t$
$T$
$T_{w}$
$u, v$
$w$
$x, y$
$z$

aspect ratios
heat capacity
gravity
cavity height
thermal conductivity
permeability
Nussely number
pressure
rate of heat generation
Darcy-Rayleigh number
time
temperature
boundary temperature
horizontal velocities
vertical velocity
horizontal coordinates
vertical coordinate

\begin{tabular}{ll}
\multicolumn{2}{l}{ Greek symbols } \\
$\alpha$ & thermal diffusivity \\
$\beta$ & thermal expansion coefficient \\
$\theta$ & temperature \\
$\theta^{n}$ & temperature at $n^{\text {th }}$ timestep \\
$\sigma$ & heat capacity ratio \\
$\mu$ & dynamic viscosity \\
$\rho$ & density \\
$\psi$ & velocity potential
\end{tabular}

Subscripts and superscripts

$(1),(2),(3) \quad$ denoting components

$c$

$\mathrm{f}$ critical value

$\max$ maximum value

pm porous medium

rms root mean square

ref reference value

dimensional 


\section{GOVERNING EQUATIONS}

A cuboidal box with dimensions, $A_{x} h, A_{y} h$ and $h$ in the $\hat{x}$-, $\hat{y}$ - and $\hat{z}$-directions is filled with a heatgenerating fluid-saturated porous medium. Here, $\hat{x}$ and $\hat{y}$ are horizontal coordinates and $\hat{z}$ is the vertical coordinate. All six walls are maintained at the constant temperature, $T_{w}$, and the saturated medium generates heat at the uniform rate, $q^{\prime \prime \prime}$. The equations for the conservation of mass, for Darcy flow, and for heat transport are as follows:

$$
\begin{gathered}
\frac{\partial \hat{u}}{\partial \hat{x}}+\frac{\partial \hat{v}}{\partial \hat{y}}+\frac{\partial \hat{w}}{\partial \hat{z}}=0, \\
\hat{u}=-\frac{K}{\mu} \frac{\partial \hat{p}}{\partial \hat{x}}, \\
\hat{v}=-\frac{K}{\mu} \frac{\partial \hat{p}}{\partial \hat{y}}, \\
\hat{w}=-\frac{K}{\mu}\left(\frac{\partial \hat{p}}{\partial \hat{z}}-\rho g \beta\left(T-T_{w}\right)\right), \\
\sigma \frac{\partial T}{\partial \hat{t}}+\hat{u} \frac{\partial T}{\partial \hat{x}}+\hat{v} \frac{\partial T}{\partial \hat{y}}+\hat{w} \frac{\partial T}{\partial \hat{z}}=\alpha\left(\frac{\partial^{2} T}{\partial \hat{x}^{2}}+\frac{\partial^{2} T}{\partial \hat{y}^{2}}+\frac{\partial^{2} T}{\partial \hat{z}^{2}}\right)+\frac{q^{\prime \prime \prime}}{(\rho C)_{f}} .
\end{gathered}
$$

In these equations $\sigma=(\rho C)_{\mathrm{pm}} /(\rho C)_{\mathrm{f}}$ is the ratio of the heat capacities of the porous medium and the saturating fluid, and $\alpha=k_{\mathrm{pm}} /(\rho C)_{\mathrm{f}}$ is a thermal diffusivity. The boundary conditions are that the normal velocities are equal to zero and that $T=T_{w}$ on all six faces of the cuboid. The height of the cuboid is taken to be $h$.

We may nondimensionalize by means of the following substitutions,

$$
\hat{t}=\frac{h^{2} \sigma}{\alpha_{\mathrm{pm}}} t, \quad(\hat{x}, \hat{y}, \hat{z})=h(x, y, z), \quad(\hat{u}, \hat{v}, \hat{w})=\frac{\alpha_{\mathrm{pm}}}{h}(u, v, w), \quad \hat{p}=\frac{\mu \alpha_{\mathrm{pm}}}{K} p, \quad T=T_{w}+\frac{q^{\prime \prime \prime} h^{2}}{k_{\mathrm{pm}}} \theta .
$$

In this way the governing equations become,

$$
\begin{gathered}
\frac{\partial u}{\partial x}+\frac{\partial v}{\partial y}+\frac{\partial w}{\partial z}=0 \\
u=-\frac{\partial p}{\partial x} \\
v=-\frac{\partial p}{\partial y}, \\
w=-\frac{\partial p}{\partial z}+\operatorname{Ra} \theta \\
\frac{\partial \theta}{\partial t}+u \frac{\partial \theta}{\partial x}+v \frac{\partial \theta}{\partial y}+w \frac{\partial \theta}{\partial z}=\frac{\partial^{2} \theta}{\partial x^{2}}+\frac{\partial^{2} \theta}{\partial y^{2}}+\frac{\partial^{2} \theta}{\partial z^{2}}+1 .
\end{gathered}
$$

The boundary conditions are now,

$$
\begin{aligned}
& u=0, \quad \theta=0, \quad \text { on } \quad x=0, A_{x}, \\
& v=0, \quad \theta=0, \quad \text { on } \quad y=0, A_{y}, \\
& w=0, \quad \theta=0, \quad \text { on } \quad z=0,1 .
\end{aligned}
$$


We now follow the methodology of Hirasaki and Hellums [1968] as modified for convection in porous media by Holst and Aziz [1972] by introducing a vector potential formulation. We may write it the form,

$$
\left(\begin{array}{l}
u \\
v \\
w
\end{array}\right)=\nabla \times\left(\begin{array}{l}
\psi^{(1)} \\
\psi^{(2)} \\
\psi^{(3)}
\end{array}\right)=\left(\begin{array}{l}
\psi_{y}^{(3)}-\psi_{z}^{(2)} \\
\psi_{z}^{(1)}-\psi_{x}^{(3)} \\
\psi_{x}^{(2)}-\psi_{y}^{(1)}
\end{array}\right),
$$

which, when it is (i) substituted into Eqs. (7) to (10), (ii) the pressure terms are eliminated by crossdifferentiation, and (iii) the vector potential is taken to be solenoidal (divergence-free), yields the following system,

$$
\begin{gathered}
\nabla^{2} \psi^{(1)}=-\operatorname{Ra} \frac{\partial \theta}{\partial y}, \\
\nabla^{2} \psi^{(2)}=\operatorname{Ra} \frac{\partial \theta}{\partial x}, \\
\nabla^{2} \psi^{(3)}=0 .
\end{gathered}
$$

The boundary conditions for all three components of the velocity potential are homogeneous, as shown below, and therefore we may conclude that $\psi^{(3)}=0$. Consequently, the heat transport equation takes the form,

$$
\frac{\partial \theta}{\partial t}+\left(\frac{\partial \psi^{(1)}}{\partial z} \frac{\partial \theta}{\partial y}-\frac{\partial \psi^{(1)}}{\partial y} \frac{\partial \theta}{\partial z}\right)+\left(\frac{\partial \psi^{(2)}}{\partial x} \frac{\partial \theta}{\partial z}-\frac{\partial \psi^{(2)}}{\partial z} \frac{\partial \theta}{\partial x}\right)=\nabla^{2} \theta+1,
$$

whie the boundary conditions transform to,

$$
\begin{array}{ccc}
\frac{\partial \psi^{(1)}}{\partial x}=0, \quad \psi^{(2)}=0, & \theta=0, & \text { on } \quad x=0, A_{x} \\
\psi^{(1)}=0, \quad \frac{\partial \psi^{(2)}}{\partial y}=0, & \theta=0, & \text { on } \quad y=0, A_{y}, \\
\psi^{(1)}=0, \quad \psi^{(2)}=0, \quad \theta=0, & \text { on } \quad z=0,1 .
\end{array}
$$

\section{NUMERICAL METHODS}

The system of partial differential equations given by Eqs. (16), (17) and (19) and which are subject to the boundary conditions, Eqs. (20) to (22), were solved numerically using a finite difference method on a uniform grid in all three coordinate directions. Both steady and unsteady solvers were created.

For the steady solver we employed a standard Full Approximation Scheme multigrid method with Vcycling in order to iterate towards solutions of the full nonlinear governing system. The basic smoother was a pointwise Gauss-Seidel iteration scheme. The nonlinear terms in the heat transport equation were approximated using Arakawa's [1966] approximation which is particularly suitable for problems involving instabilities. The Neumann derivatives in Eqs. (20) and (21) were approximated using the fictitious point technique, thereby maintaining second order accuracy in space. Convergence was deemed to have taken place once the residual had fallen below $10^{-8}$.

Two different unsteady solvers were written. The first employed the du-Fort-Frankel method to update the temperature field at each timestep, while the two Poisson equations, (16) and (17), were solved using the correction scheme multigrid method with V-cycling where the smoother was a line relaxation Gauss-Seidel solver in all three coordinate directions. Formally this type of timestepping has second order accuracy in time, and although it is formally declared as being unconditionally stable when solving a Poisson's equation, it nevertheless yields unphysical oscillations from time step to time 
step, and this restricts the range of allowable time steps. The second method was a straightforward modification of the steady solver described above, and therefore it was a fully implicit time stepping scheme. In what follows, we do not mention which unsteady solver was used because all flows tended towards a steady state when $R a \leq 6000$. All the codes were given options which allowed various symmetries in the solutions to be maintained, should that be necessary. For convergence to the steady state we required that the following condition is satisfied,

$$
\left|\frac{\theta_{\max }^{n+1}-\theta_{\max }^{n}}{\theta_{\max }^{n+1}}\right|<10^{-8} .
$$

\section{RESULTS AND DISCUSSION}

In this paper we have restricted our attention to a $2 \times 2 \times 1$ cuboid, i.e. one with a unit height and a square planform, and thus $A_{x}=A_{y}=2$ in Eqs. (12) and (13). Additionally, we have confined computations to values of the Darcy-Rayleigh number which are at or below 6000. The dimensions of the cuboid are such that equal steplengths in all three directions have been taken. This self-imposed limit on the Darcy-Rayleigh number means that the flow always evolves to the basic state.

\section{The primary convection pattern}

When the Darcy-Rayleigh number is zero the temperature field will satisfy a Poisson's equation of the form, $\nabla^{2} \theta=-1$, and, given that the six bounding faces are subject to the boundary condition, $\theta=0$, the resulting temperature field is essentially symmetric about all three mid-planes and takes its maximum value at the precise centre of the cavity, namely, $x=y=1$ and $z=\frac{1}{2}$, at which point $\theta=0.106157$. Of more interest is the RMS value of $\theta$ :

$$
\theta_{\mathrm{rms}}=\sqrt{\frac{1}{4} \int_{0}^{2} \int_{0}^{2} \int_{0}^{1}[\theta(x, y, z)]^{2} d x d y d z .}
$$

For this conduction problem we have,

$$
\theta_{\mathrm{rms}}=5.049917 \times 10^{-2} .
$$

This value was obtained from the triple half-range Fourier series solution,

$$
\theta=\frac{256}{\pi^{5}} \sum_{\substack{n_{1}=1 \\\left(n_{1} \text { odd }\right)}}^{\infty} \sum_{\substack{n_{2}=1 \\\left(n_{2} \text { odd }\right)\left(n_{3} \text { odd }\right)}}^{\infty} \sum_{n_{3}=1}^{\infty} \frac{\sin \frac{n_{1} \pi x}{2} \sin \frac{n_{2} \pi y}{2} \sin n_{3} \pi z}{n_{1} n_{2} n_{3}\left(n_{1}^{2}+n_{2}^{2}+4 n_{3}^{2}\right)}
$$

At small values of $R a$ a weak circulation is induced with up-welling in the middle of the cavity and down-welling next to the vertical walls. Thus a toroidal circulation is set up naturally because of the presence of the cold sidewalls. Figure $1 \mathrm{~b}$ illustrates this by showing the velocity vectors along the upper surface for the moderate case, $R a=500$. By contrast, a layer of infinite extent has no such circulation. If we define the local Nusselt number as being the rate of heat transfer at the upper surface,

$$
N u(x, y)=-\left.\frac{\partial \theta}{\partial z}\right|_{z=1},
$$

then it is seen to vary from zero at the sidewalls to a maximum in the middle, as also depicted in Fig. 1a. Figure $1 \mathrm{c}$ also shows the temperature contours in the $(x, z)$-plane at $y=1$ (or equivalently, in the $(y, z)$ plane at $x=1$ ). The maximum value of $\theta$ is computed to be, $\theta_{\max }=0.08762$, which is a little less than 

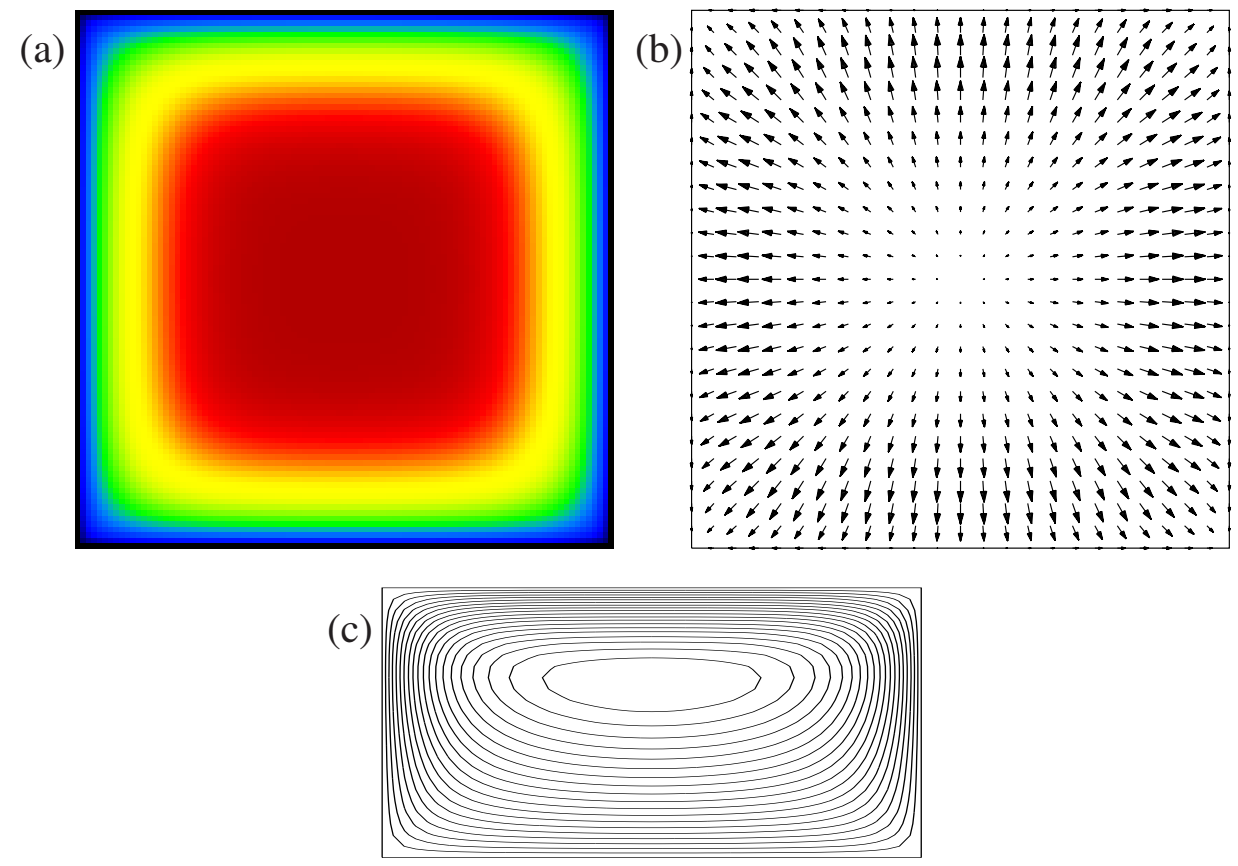

Figure 1. Depicting the upper surface (a) Nusselt number and (b) velocity vectors, and (c) the mid-plane $(y=1)$ temperature contours for $R a=500$ for the $\square$-pattern. Frames (a) and (b) occupy $0 \leq x \leq 2$ and $0 \leq y \leq 2$, while frame (c) occupies $0 \leq x \leq 2$ and $0 \leq z \leq 1$.

the value given above for the conduction solution at $R a=0$. The position of this maximum has risen slightly from its previous position at the centre of the cavity.

As $R a$ increases further, the circulation grows in strength and the temperature field, as represented by $N u$, tends to become more uniform in the centre $(x \simeq 1, y \simeq 1)$. This is depicted in Fig. 2 for $R a=5000$, together with the corresponding upper surface velocity vectors. The maximum temperature has now reduced again to $\theta_{\max }=0.04646$, and its location has risen further. The red band in Fig. 2a indicates the locus of the largest rate of heat transfer, and this is to be contrasted with Fig. 1a, where the maximum is in the centre of the upper surface. Taken together, all three frames of Fig. 2 show that boundary layers have begun to form on the sidewalls and the upper surface as $R a$ increases. The isotherms in Fig. (2c) also show that the chosen grid is approaching its limit in being able to resolve the flows well.

The flow patterns which are shown in Figs. 1 and 2 may be regarded as being the basic state whose stability and non-uniqueness is the subject of this paper. For the sake of both brevity and clarity we shall also refer to it as the $\square$-pattern, given the shape of the pattern shown in Figs. (1a) and (2a).

Figure (2c) exhibits a fairly thin region near the upper surface where hot fluid lies below cold fluid. This is the classic situation for which a Darcy-Bénard instability might occur. For the case shown in Fig. (2c) one may estimate the local Darcy-Rayleigh number from the maximum temperature and the thickness of the layer; we find that it is approximately 46, which we obtained by multiplying the overall value for $R a$ by the maximum temperature and the relative depth of the thermal boundary layer. This is very close to the value of $4 \pi^{2}$ which corresponds to the Darcy-Bénard problem with impermeable boundaries, and doesn't suggest that one may have strong convection at this value of $R a$. Perhaps a better comparison, though, might be with the Wooding problem, a region of fluid-saturated porous media which is heated from below by a uniform constant temperature and where the lower surface boundary is subject to uniform suction. In the deep-pool version of the Wooding problem (where there is no upper surface but where the uniform suction confines the thermal boundary layer to the vicinity of the suction surface) the critical value of the Darcy-Rayleigh number is now 14.3552 (Rees [2009]). The implication 

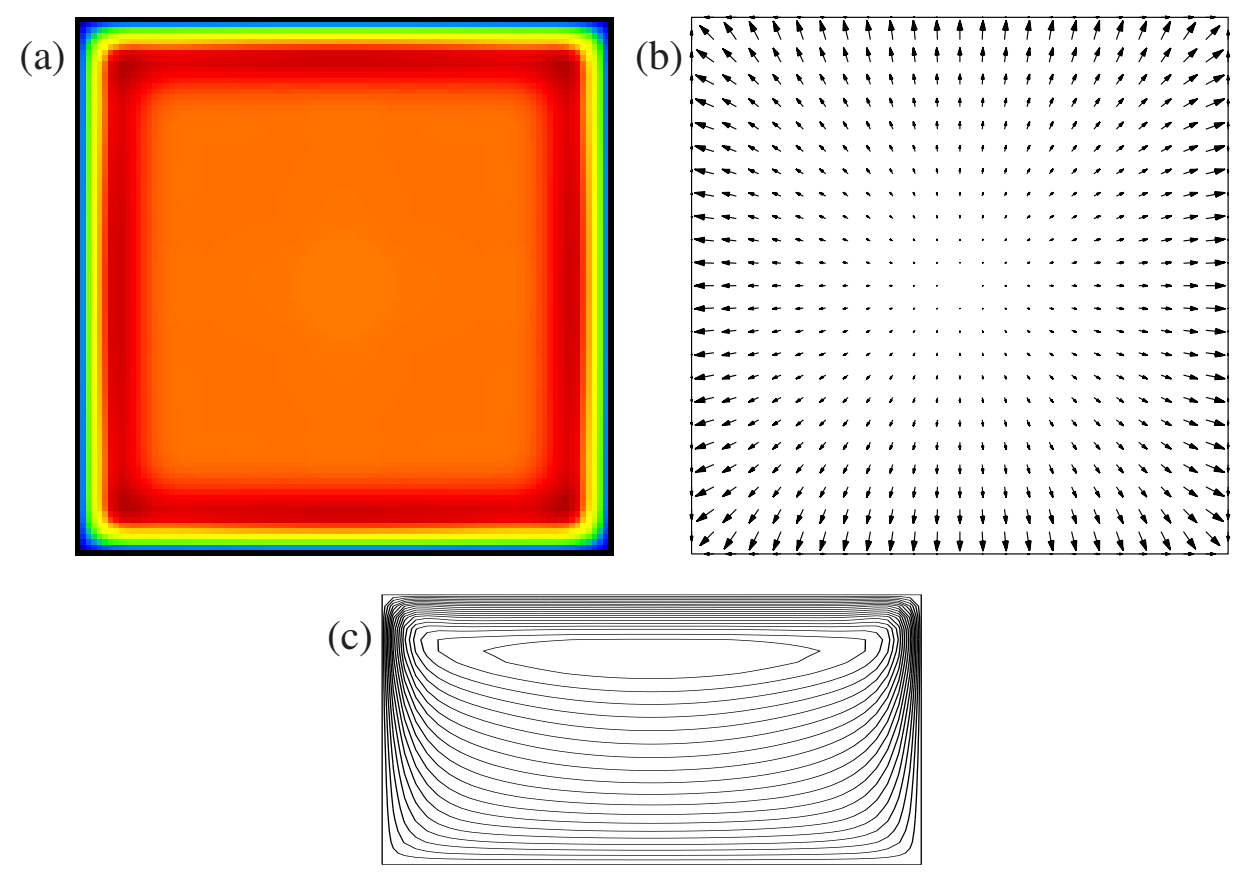

Figure 2. Depicting the upper surface (a) Nusselt number and (b) velocity vectors, and (c) the mid-plane $(y=1)$ temperature contours for $R a=5000$ for the $\square$-pattern.

now is that $R a=5000$ could well represent a strongly supercritical configuration with different flow patterns arising at smaller values of $R a$.

\section{Inventory of possible steady solutions}

In our unsteady computations, which were undertaken with initial temperature profiles displaying a large variety of different symmetries, we have identified four more steady state flows which exist when $R a \leq 6000$; these are depicted in Figs. 3 to 6. Figure 3 displays the $\square$-pattern at $R a=5000$, and it shows a Nusselt number profile which is similar to that of the $\square$-pattern in Fig. 2 but where there is a significant change in behaviour in the middle of the upper surface. This pattern is characterised by having down-welling in the centre of the cuboid in addition to the buoyancy-induced down-welling on the sidewalls. Thus the flow is comprised of two closed-loop toroidal vortices, the outer one having fluid moving across the top of the box towards the sidewalls and the inner one having fluid moving towards the centre of the box. This pattern is seen clearly in the velocity vectors, and the temperature contours of the vertical cross-section on $y=1$ shows the corresponding downward deformation of the isotherms at the centre, and it is this which reduces the surface rate of heat transfer, as seen in Fig. 3c.

Figure 4 shows what we term the $\square$-pattern. Once more there is downwelling at the centre of the cuboid, but in this case the flow is almost perfectly two-dimensional in the range, $\frac{2}{3}<y<\frac{4}{3}$ (i.e. the central one third) and has overtly three-dimensional behaviour elsewhere. An alternative way of thinking about this is, upon referring to the velocity vectors, that the cavity is split into four cells whose axes are roughly in the $y$-direction. The temperature contours at $y=1$ are almost identical to those shown in Fig. 3c, even though the overall pattern of convection displays a slightly different set of symmetries. We note that there is also a $\boxminus$-pattern which is identical in all respects but for a rotation though $90^{\circ}$ — this is precisely the same convection pattern given that the cavity has a square planform.

Figure 5 shows the $\square$-pattern which is very similar to the $\square$-pattern in that the cavity is split into four convection cells. However, the central down-welling which is a feature of both the $\square$ and $\square$ patterns is 

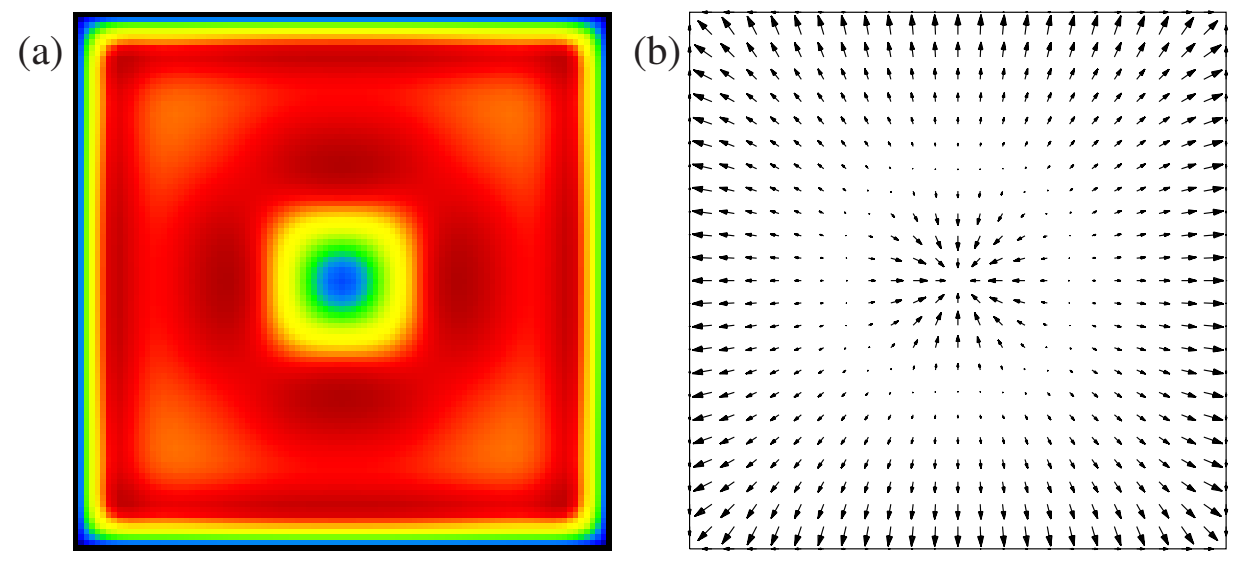

(c)

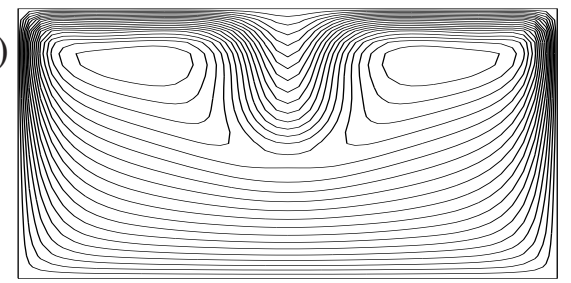

Figure 3. Depicting the upper surface (a) Nusselt number and (b) velocity vectors, and (c) the mid-plane $(y=1)$ temperature contours for $R a=5000$ for the $\square$-pattern.
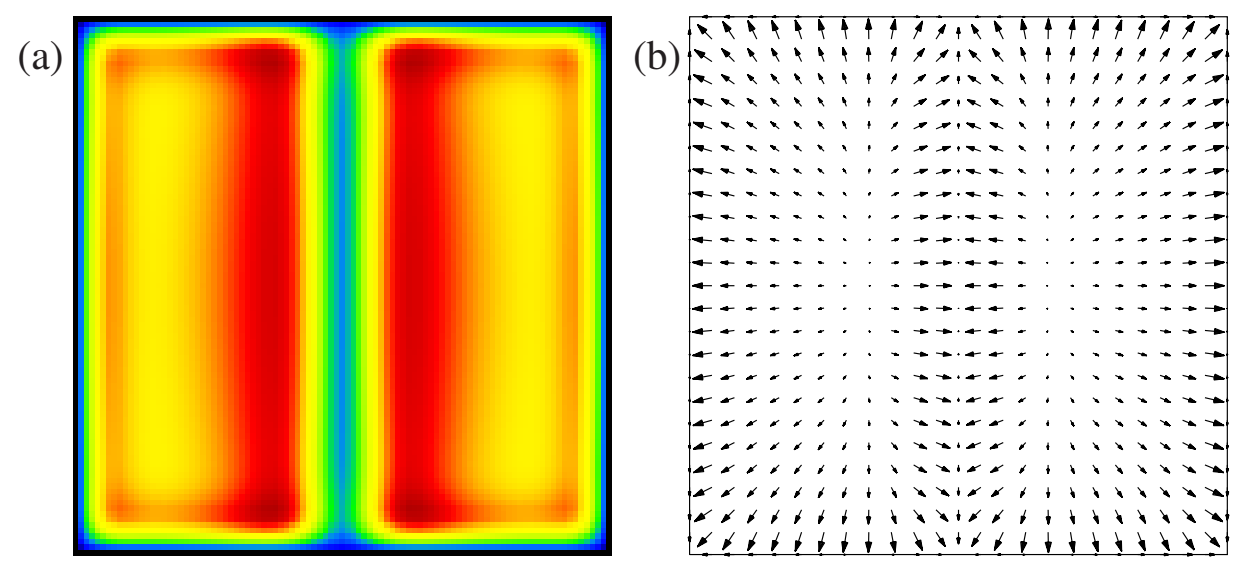

(c)

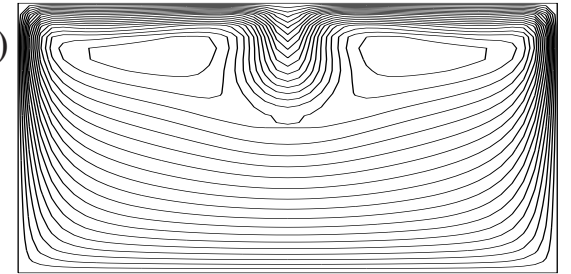

Figure 4. Depicting the upper surface (a) Nusselt number and (b) velocity vectors, and (c) the mid-plane $(y=1)$ temperature contours for $R a=6000$ for the $\square$-pattern. 

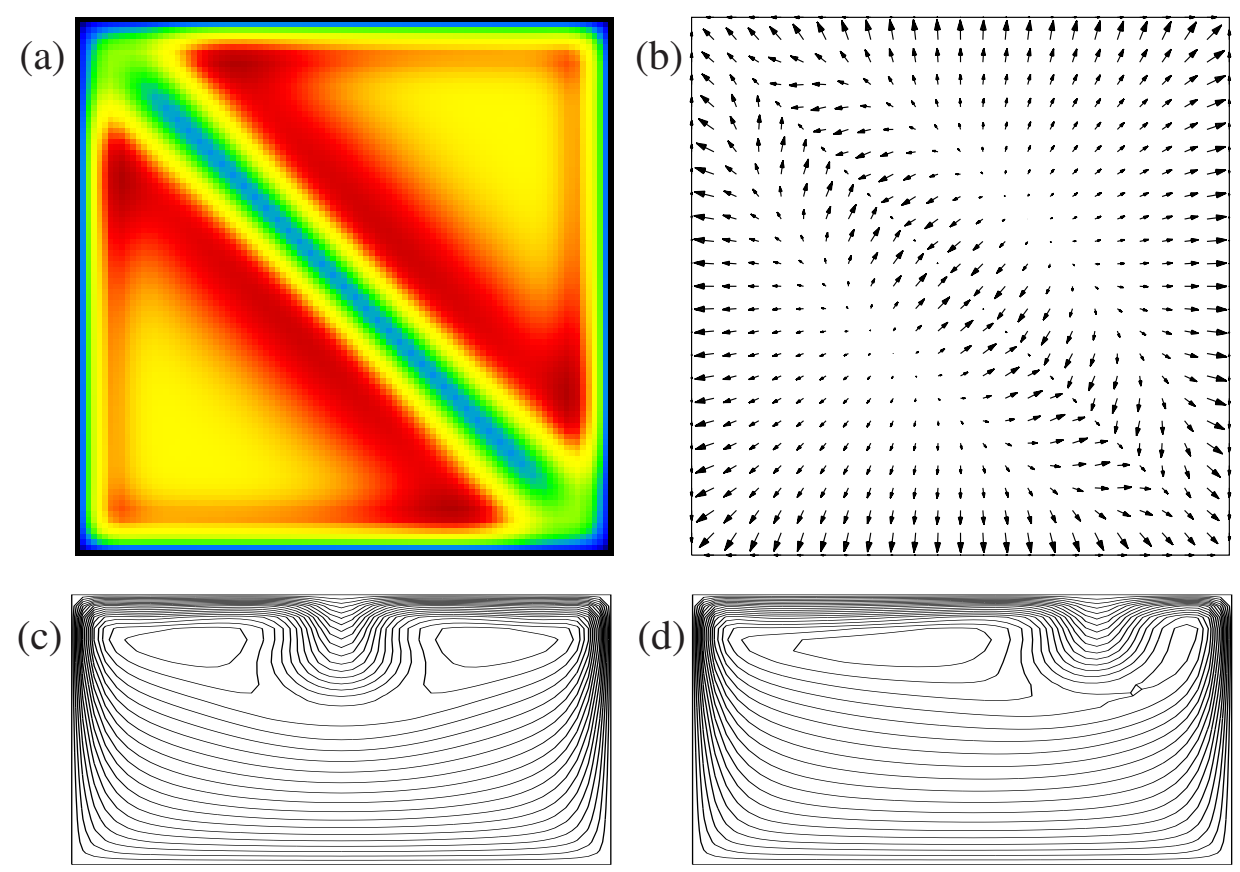

Figure 5. Depicting the upper surface (a) Nusselt number and (b) velocity vectors, the (c) mid-plane $(y=1)$ temperature contours and the (d) quarter-plane $\left(y=\frac{1}{2}\right)$ temperature contours for $R a=6000$ and for the $\nabla$-pattern.

now located along one of the two diagonals of the top surface, and there are two narrow vortices placed immediately either side of this diagonal. The strength of the down-welling along the diagonal varies little, as may be inferred from a comparison of Figs. $5 \mathrm{c}$ and $5 \mathrm{~d}$, which correspond to the isotherms along $y=1$ and $y=\frac{1}{2}$. Clearly there also exists what may be called the $\square$-pattern which is the mirror image of this one, but it is otherwise identical.

Finally, in our inventory of possible convection patterns, there is the $\otimes$-pattern which may be regarded as being composed of equal amounts of the $\square$ and $\square$ patterns. This is shown in Fig. 6. The upper-surface velocity vectors show that there are five stagnation points on the upper surface, four of which correspond to up-welling. The fifth is at the centre of the cavity and corresponds once more to down-welling. But given that this pattern is composed of a 'sum' of two other patterns, it might be thought that a $\boxplus$-pattern might be composed of a superposition of the $\square$ and $\boxminus$ patterns. This has been searched for extensively but not found, although this doesn't preclude the possibility of it not existing when $R a>6000$.

\section{The solution branches}

Having established that there are five steady-state patterns of convection in a $2 \times 2 \times 1$ cavity when $R a \leq 6000$, the next questions concern how they are related and whether a bifurcation diagram may be constructed. The answers begin with the solution curves shown in Figs. 7 and 8, which show our computed values of $\theta_{\mathrm{rms}}$ for each pattern and their variation with $R a$. One may use other measures of the strength of the flow, such as the mean rate of heat transfer through the upper surface, the maximum temperature and the heat transfer at the middle of the upper surface, and it is possible that these may yield further insights in future work.

Only one curve begins at $R a=0$ and this corresponds to the $\square$-pattern shown in Figs. 1 and 2 . The curve was obtained by using the steady-state code as described earlier, where the initial condition for each value of $R a$ was the final steady solution from the previous computation. We were able to 
(a)

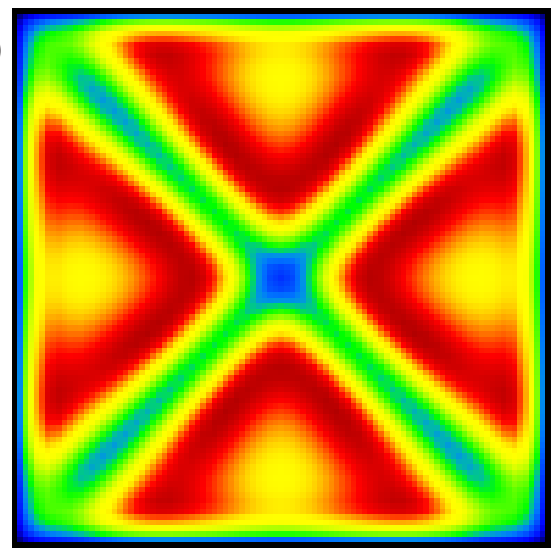

(c)

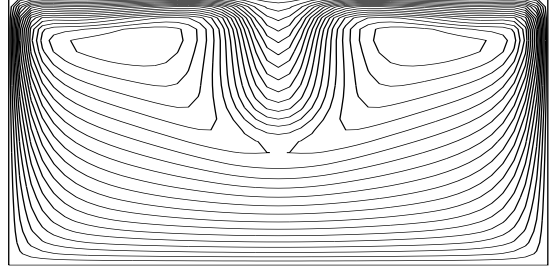

(b)

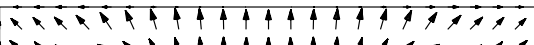

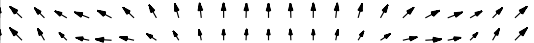

$\rightarrow 1+\ldots \ldots,+1,1, \rightarrow \rightarrow+1$

B

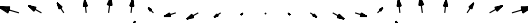

ant

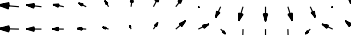

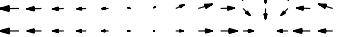

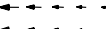

$\therefore \therefore \rightarrow+1+2$

-

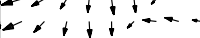

r,

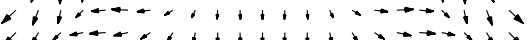

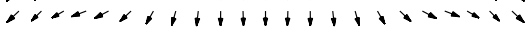

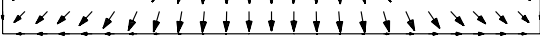

(d)

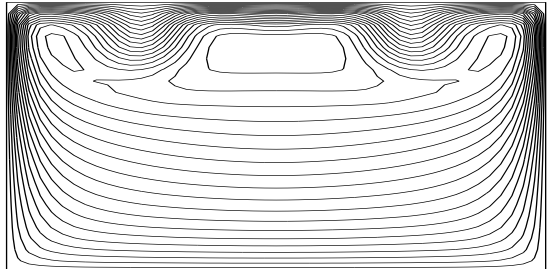

Figure 6. Depicting the upper surface (a) Nusselt number and (b) velocity vectors, the (c) mid-plane $(y=1)$ temperature contours and the (d) quarter-plane $\left(y=\frac{1}{2}\right)$ temperature contours for $R a=6000$ and for the $\otimes$-pattern.

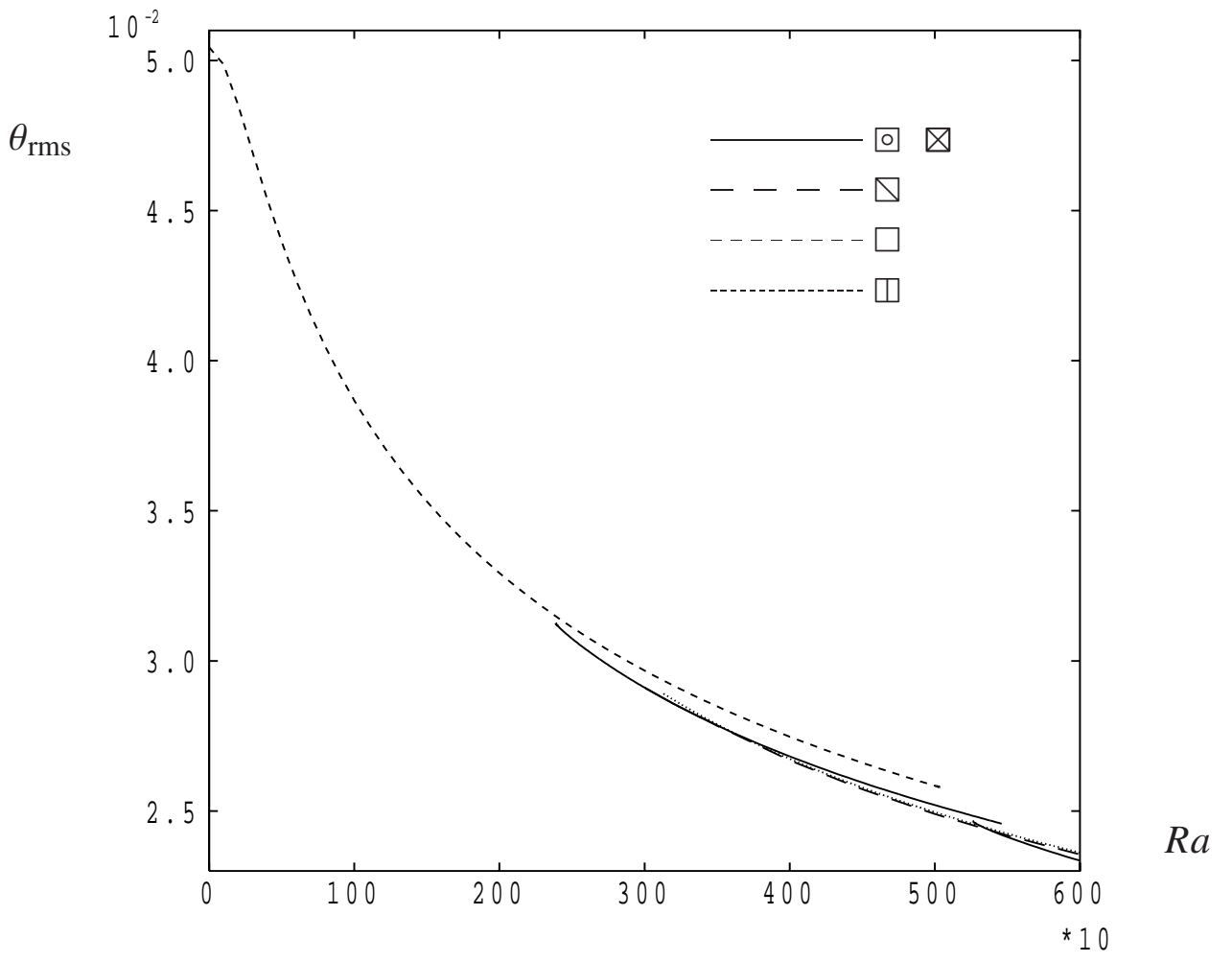

Figure 7. The variation with $R a$ of $\theta_{\text {rms }}$ for each of the five convection patterns. 


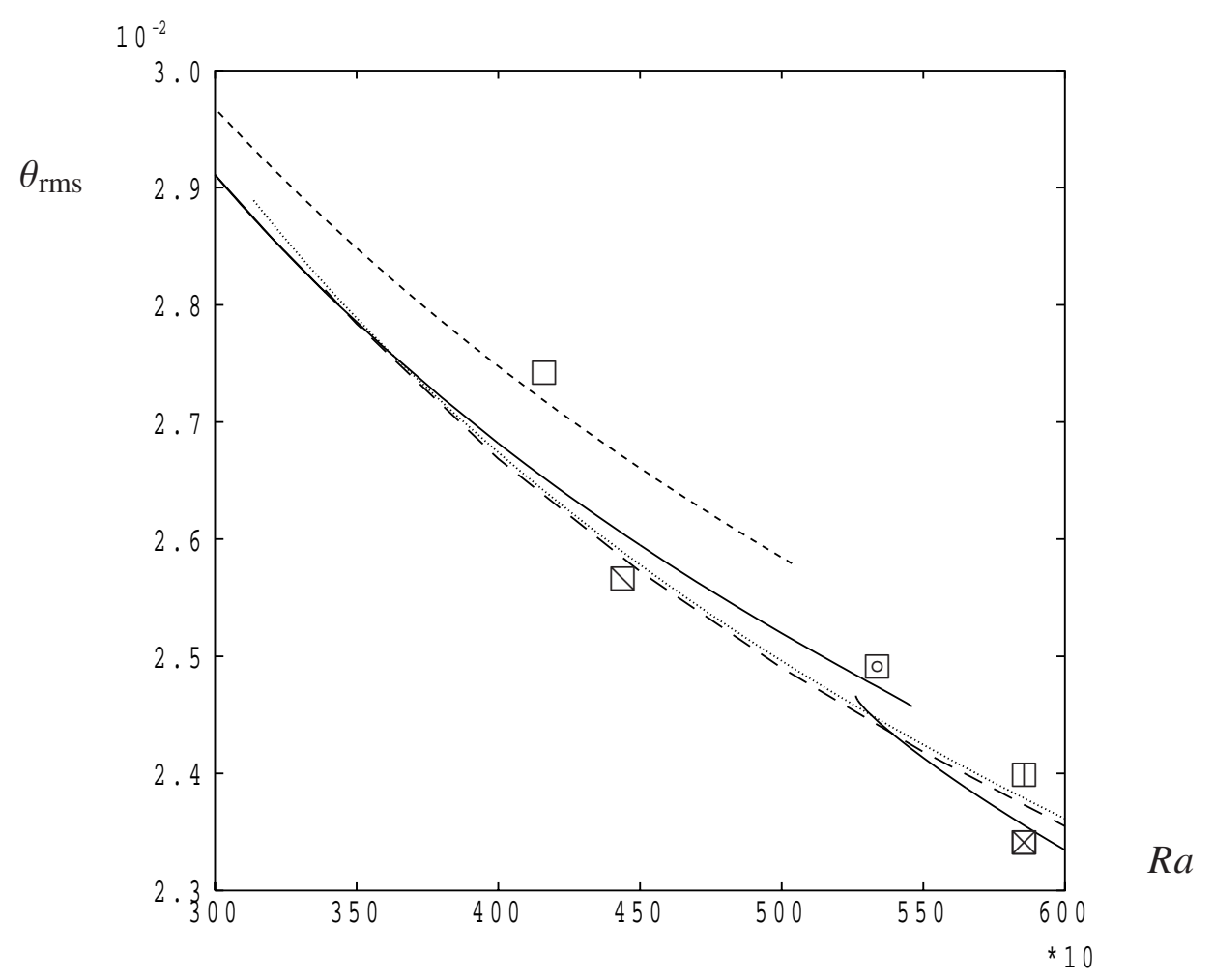

Figure 8. A close-up view of the curves shown in Fig. 7 in the range, $3000 \leq R a \leq 6000$.

constrain solutions for $\theta$ to those which maintained even symmetry about $x=1$ and $y=1$. In this way it was possible to obtain converged solutions in the range $0 \leq R a \leq 5036$. This upper limit was characterized by an increasing number of iterations to attain steady state. This may be a sign of two potential difficulties: (i) the increasing effect of nonlinearity and (ii) the proximity of a bifurcation both of these features are well-known causes for convergence difficulties. This value of $R a$ is already well into the regime within which instability could be expected and therefore the unsteady code was used to perturb many of the steady-state solutions on this curve. The rather surprising result of this numerical experiment was that we found the curve to represent a state which is linearly stable along the whole of the length plotted in Fig 7; instability could be induced but only with disturbances of moderate amplitude. Given that we were able to find steady solutions with other patterns at larger values of $R a$, we conclude that the termination of this $\square$-branch is not due to the nonlinearity being too strong, but because of either a bifurcation or a turning point.

We now focus on the branch corresponding to the $\square$-pattern. This is the continuous line lying in the range below approximately $R a=5500$. In Fig. 7 it terminates just below $R a=2387$ without attaching to the $\square$-branch. Using the maximum value of $\theta$ as a measure (not shown here) the curve very clearly ends at a very distinct turning point, and therefore our best guess, in the absence of further solutions (which are very likely to be unstable) is that this solution branch turns back in the direction of increasing values of $R a$. Our suspicion is that that branch will eventually attach to the branch of the $\square$-pattern very close to $R a=5036$. The reason for this conclusion is that the $\square$ and $\square$ patterns share all their symmetries (i.e. mirror symmetry about both $x=1$ and $y=1$, and about the two main diagonals). If this reasoning is correct, then the apparent termination of the $\square$-branch at $R a=5036$ is not a bifurcation but another turning point. At present this is our best guess because a bifurcation always involves the breaking of at least one symmetry. So it is our belief that the $\square$ and $\square$ branches form a single branch and are joined by means of two turning points and a joining intermediate solution branch.

Generally, if an unsteady computation is undertaken with $\theta=0$ as the initial condition, then if 
$R a<3500$ then the steady solution which is obtained depends on whether $R a$ is above 2387 or not. So it appears that the $\square$-pattern is the favoured pattern at least for a range of values of $R a$ above 2387 .

When $R a$ rises above about 3500 the $\square$-pattern is usually favoured when using an unsteady solver. Thus it seems that that that curve with the smallest value of $\theta_{\text {rms }}$ may be regarded as being a strong candidate for being the favoured mode. In a sense, this is reminiscent of the Malkus and Veronis maximum heat transfer hypothesis (which is approximately true at best) for the selection of the most likely mode of convection in the Bénard problem. In the Bénard problem there is a fixed temperature drop across the layer and maximising the heat transfer was said to provide a selection criterion for the most likely mode to appear. Here the rate of heat transfer is specified and therefore the equivalent hypothesis would be that the most favoured mode minimises the maximum temperature or the value of $\theta_{\text {rms }}$. That said, we think it likely that both the $\square$ and $\square$ patterns are realiizable for a range of values of $R a$ near to where the corresponding solution curves cross, although we have not yet undertaken a detailed study of this aspect.

We now refer to Fig. 8 in order to see more detail in the curves when $R a$ is close to 6000 . The branch corresponding to the $\nabla$-pattern may now be computed all the way on to $R a=6000$ using the steady solver, although it ceases to be linearly stable near to $R a=6000$. At this far end of our chosen range for the Darcy-Rayleigh number the $\otimes$-pattern makes its appearance. Whilst it looks as though it is a superposition of the $\square$ and $\square$ patterns, it nevertheless shares all the of the symmetries satisfied by the $\square$ and $\square$ patterns. The $\otimes$-pattern always arises when using an unsteady solver when $R a>5500$, but as $R a$ decreases towards 5281 the number of iterations required for convergence of the steady solver increases greatly. This also happens for the branch corresponding to the $\square$-pattern as $R a$ approaches 5460 from below. Given that these patterns share all their symmetries and that there are no other solution branches available, it seems very likely that the we again have a pair of turning points and that the free ends of the two continuous curves shown in Fig. 8 are joined by another curve. Therefore we have a continuous transition from a $\square$-pattern via the $\square$-pattern to the $\otimes$-pattern which traverses three branches and four turning points.

The $\square$-pattern curve may be computed using the steady solver from $R a=6000$ down to $R a=3130$. Once more the number of iterations increases greatly, and therefore we have to conclude that the curve turns back towards higher values of $R a$, as did the $\square$-branch. At present we do not know the ultimate fate of such a branch. However, any unsteady siulations that we have run using an initial condition which looks like the $\square$-pattern inevitably evolves towards either the $\square$ or the $\nabla$ pattern, depending on the value of $R a$. Therefore while this is a potential nonlinear steady solution, it is one which is always unstable.

\section{Comparison with 2D cavities}

It is worth considering how the above scenario compares with the two-dimensional simulations in Banu [2000]. For a $2 \times 1$ cavity the temperature field looks very much like that shown in Figs. (1c) and (2c) when $R a$ takes small values. As $R a$ increases there is a smooth transition to a pattern like that shown in Fig. (3c), unlike for the three-dimensional cavity where such a state is approached via two turning points in the solution curve. The two-dimensional cavity also admits unsteady solutions when $R a$ is as low as 2200; this unsteady convection pattern arises when the downward-facing plume in the centre of cavity (see Fig. (2c)) is perturbed slightly to one side. The common effect of a neighbouring wall drawing a plume towards itself due to unequal entrainment (Brambles and Rees [2007]) then causes the plume to migrate further away from the centre. This leaves a very large region of thermal boundary layer at the upper surface which is potentially thermoconvectively unstable. A new plume is then generated on the opposite side of the centre of the cavity which grows subsequently and eventually migrates in the opposite direction to that of the original plume. The whole system soon settles into one which is timeperiodic and which is characterised by a plume being generated and moving in the opposite direction to its predecessor. In the present three-dimensional context the presence of four sidewalls clearly delays the onset of unsteady convection to much higher values of $R a$. 


\section{CONCLUSIONS}

In this paper we have presented some three-dimensional computations of convection inside a cuboidal porous cavity subjected to internal heating. We have restricted our attention to a $2 \times 2 \times 1$ cavity for values of the Darcy-Rayleigh number which are below 6000. While this seems at first glance to be quite restrictive, we have found five different convection patterns and have made various detailed inferences based on the solution curves and the symmetries of the respective convection patterns. It is clear that the two-dimensional computations of Banu [2000] and the present three-dimensional computations differ greatly in terms of the qualitative nature of the solutions; this is due to the fact that Dirichlet conditions on all four vertical walls induce three-dimensional flows at all non-zero values of the Darcy-Rayleigh number. For example, persistently unsteady two-dimensional convection arises when $R a$ is near to 2200 in a $2 \times 1$ cavity, whereas it has to be in excess of 6000 for three-dimensional convection.

\section{REFERENCES}

Arakawa, A. [1966], Computational study of long-term numercal integration of the equations of fluid motion: two-dimensional incompressible flow, J. Comp. Phys., Vol. 143, pp. 1-119.

Banu, N. [2000], Convection in Porous Media, Ph.D. Thesis, Dept. of Mech. Eng., University of Bath, UK.

Banu, N., Rees, D.A.S., Pop, I. [1998], Steady and unsteady convection in rectangular porous cavities with internal heat generation, Proc. 11th Int. Heat Transfer Conference (August 1998 Kyongju, Korea), Vol. 4, pp. 375-380.

Bhadauria, B.S., Hashim, I., Siddheshwar, P.G. [2013], Study of heat transport in a porous medium under g-jitter and internal heating effects, Transport in Porous Media, Vol. 96, pp. 21-37.

Blythe, P.A., Daniels, P.G., Simpkins, P.G. [1985], Convection in a fluid-saturated porous medium due to internal heat generation, Int. Comm. Heat Mass Transfer, Vol. 12, pp. 493-504.

Brambles, O.J., Rees, D.A.S. [2007], Curved free convection plume paths in porous media, Idöjárás. Quarterly Journal of the Hungarian Meteorological Service, Vol. 111, pp. 109-121.

Buretta, R.J., Berman, A.S. [1976], Convection heat transfer in a liquid saturated porous layer, A.S.M.E. J. Appl. Mech., Vol. 43, pp. 249-253.

Gasser, R.D., Kazimi, M.S. [1976], Onset of convection in a porous medium with internal heat generation, A.S.M.E. J. Heat Transfer, Vol. 98, pp. 49-54.

Hirasaki, G.J., Hellums, J.D. [1968], A general formulation of the boundary conditions on the vector potential in threedimensional hydrodynamics, Qaurt. Appl. Math., Vol. 16, pp. 331-342.

Holst, P.H., Aziz, K. [1972], Transient three-dimensional natural convection in confined porous media, Int. J. Heat Mass Transfer, Vol. 15, pp. 73-90.

Malkus, W.V.R., Veronis, G. [1958], Finite amplitude cellular convection, J. Fluid Mech., Vol. 4, pp. $225-260$.

Mealey, L.R., Merkin, J.H. [2009], Steady finite Rayleigh number convective flows in a porous medium with internal heat generation, Int. J. Thermal Sci., Vol. 48, pp. 1068-1080.

Nield, D.A., Kuznetsov, A.V. [2013], Onset of convection with internal heating in a porous medium saturated by a nanofluid, Transport in Porous Media, Vol. 99, pp. 73-83.

Nouri-Borujerdi, A., Noghrehabadi, A.R., Rees, D.A.S [2007], The onset of convection in a horizontal porous layer with uniform heat generation using a thermal non-equilibrium model, Transport in Porous Media, Vol. 69, pp. $343-357$.

Prasad, V. [1987], Thermal convection in a rectangular cavity filled with a heat-generating porous medium, A.S.M.E. J.Heat Transfer, Vol. 109, pp. 697-703.

Rees, D.A.S. [2009], The onset and nonlinear development of vortex instabilities in a horizontal forced convection boundary layer with uniform surface suction, Transp. Porous Media [R.A.Wooding Special Issue], Vol. 77, pp. 243-265.

Tveitereid, M [1977], Thermal convection in a horizontal porous layer with internal heat sources, Int. J. Heat Mass Transfer, Vol. 20, pp. 1045-1050.

Vasseur, P., Nguyen, I.H., Robillard, L., Thi, V.K.I. [1984], Natural convection between horizontal concentric cylinders filled with a porous layer with heat generation, Int. J. Heat Mass Transfer, Vol. 27, pp. 337-349. 